\title{
QM(DFT) and MD studies on formation mechanisms of $\mathrm{C}_{60}$ fullerenes
}

\author{
Xinlei Hua, Tahir Çağın, Jianwei Che and William A Goddard III \\ Materials and Process Simulation Center, Beckman Institute (139-74), Division of Chemistry \\ and Chemical Engineering, California Institute of Technology, Pasadena, California 91125, \\ USA \\ E-mail: tahir@wag.caltech.edu and wag@wag.caltech.edu
}

Received 2 March 2000

\begin{abstract}
One of the most puzzling aspects of fullerenes is how such complicated symmetric molecules are formed from a gas of atomic carbons, namely, the atomistic or chemical mechanisms. Are the atoms added one by one or as molecules $\left(\mathrm{C}_{2}, \mathrm{C}_{3}\right)$ ? Is there a critical nucleus beyond which formation proceeds at gas kinetic rates? What determines the balance between forming buckyballs, buckytubes, graphite and soot? The answer to these questions is extremely important in manipulating the systems to achieve particular products. A difficulty in current experiments is that the products can only be detected on time scales of microseconds long after many of the important formation steps have been completed. Consequently, it is necessary to use simulations, quantum mechanics and molecular dynamics, to determine these initial states. Experiments serve to provide the boundary conditions that severely limit the possibilities. Using quantum mechanical methods (density functional theory (DFT)) we derived a force field (MSXX FF) to describe one-dimensional (rings) and two-dimensional (fullerene) carbon molecules. Combining DFT with the MSXX FF, we calculated the energetics for the ring fusion spiral zipper (RFSZ) mechanism for formation of $\mathrm{C}_{60}$ fullerenes. Our results shows that the RFSZ mechanism is consistent with the quantum mechanics (with a slight modification for some of the intermediates).
\end{abstract}

\section{Introduction}

One of the most puzzling aspects of fullerenes $\left(\mathrm{C}_{60}, \mathrm{C}_{70}\right.$, etc $)$ is how such complicated symmetric molecules are formed from a gas of atomic carbons, namely, the atomistic or chemical mechanism. Are the atoms added one by one or as molecules $\left(\mathrm{C}_{2}, \mathrm{C}_{3}\right)$ ? Is the $\mathrm{C}_{60}$ fullerene formed by adding $\mathrm{C}_{1}, \mathrm{C}_{2}$, or $\mathrm{C}_{3}$ to some smaller fullerene or is $\mathrm{C}_{60}$ formed by isomerization of some type of precursor molecule $\mathrm{C}_{60}$ ? Is there a critical nucleus beyond which formation proceeds at gas kinetic rates? What determines the balance bwtween forming buckyballs, buckytubes, graphite, and soot? The answer to these questions might lead to means of manipulating the systems to achieve particular products.

A difficulty in current experiments (Hunter et al 1993, 1994, von Helden et al 1993a, b) is that the products can only be detected on time scales of $\mu \mathrm{s}$, long after many of the important formation steps have been completed. Consequently, it is necessary to use first principles quantum mechanical theory to determine these initial states; however, the experiments serve to provide boundary conditions that severely limit the possibilities, making the use of first principles theory practicable.

\section{Calculations}

We selected density functional theory (DFT) as the best compromise between accuracy and speed for studying these systems. We use the Becke gradient corrected exchange and the gradient corrected correlation functional of Lee, Yang, and Parr (Johnson et al 1993). The calculations were carried out using Jaguar program (PS-GVB) with the 6-31G* basis set (Rignalda et al 1995).

Because the carbon rings play a central role, we studied how the structures and energetics of such rings changed with size and extracted a force field (denoted as the MSX FF) that would reproduce the DFT energetics and structures. This MSX FF would be used later in conjunction with the DFT calculations on various multi-ring systems to estimate the energetics of the full 60-atom systems without the necessity of DFT on the complete system.

The calculations on ring systems up to $\mathrm{C}_{60}$ are shown in figure 1 . The energies quoted here are cohesive energy per carbon atom. In calculating these energies we used as our reference the triplet $\mathrm{C}$ atom, calculated by LSDA.

We found that

- For $n=4 m$, the minimum energy structure has a polyacytelene geometry of alternating single and triple bonds. The bond length difference is from 0.5 to $0.9 \AA$ A . We find that inclusion of correlation reduces the dimerization amplitude, similar to the case in 


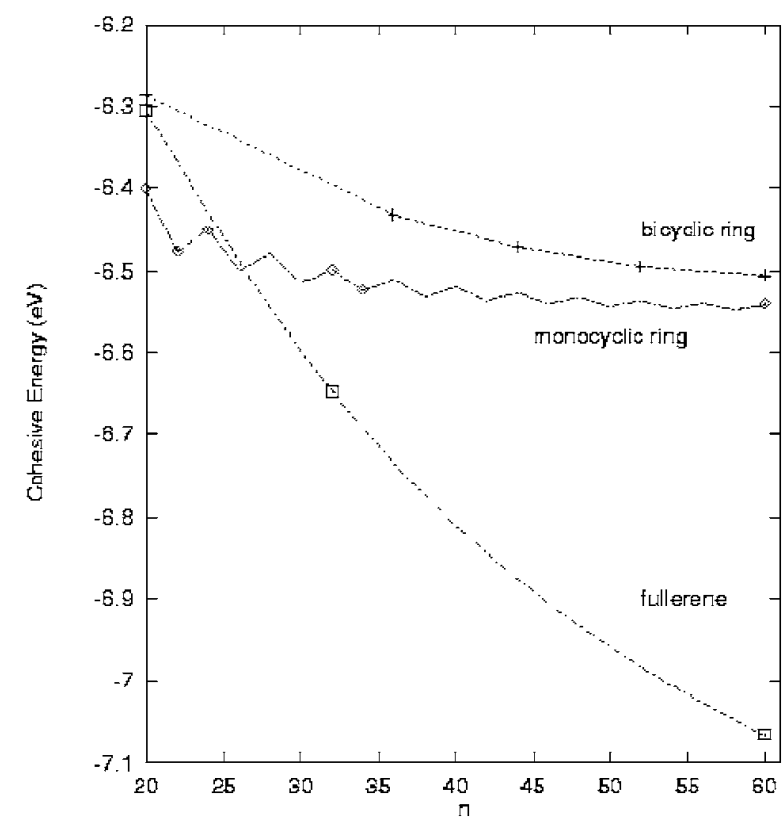

Figure 1. Cohesive energy per carbon atom of carbon clusters: monocyclic ring, bicyclic rings and fullerenes.

polyacetylene (Konig and Stollhoff 1990). Compared with the DFT geometry, Hartree-Fock (HF) gives too large a bond alternation, along with too large angle alternations. Our HF calculation gives a bond difference of $0.16 \AA$, in agreement with that of Feyereisen et al (1992). As for angle alternation, for $\mathrm{C}_{20} \mathrm{HF}$ gives 160 $164^{\circ}$ (Raghavachari et al 1993) while DFT gives 161.5$162.5^{\circ}$.

- For $n=4 m+2$, the minimum energy structure has the polyallene geometry with equal bond lengths. This is due to the resonance between the two structures, involving the $\pi$-bond perpendicular to the plane and $\pi$ bond parallel to the plane.

$\mathrm{C}_{4 m+2}$ is more stable than $\mathrm{C}_{4 m}$. But as $n \rightarrow \infty$, the difference in $E_{\text {coh }}$ decreases to zero, leading to $E_{\infty}\left(\mathrm{sp}^{1}\right)=6.56 \mathrm{eV}$. Both the polyacetylene and polyallene structures involve $\sigma$ bonds that are $\mathrm{sp}^{1}$ hybrids, which prefer linear geometries. Thus we expect a strain energy proportional to $(\delta \theta)^{2}=$ $(\pi-\theta)^{2}=(2 \pi / n)^{2}$. Indeed we found strain energy increase linearly with $1 / n^{2}$ with slopes of $63.3 \mathrm{eV} / n^{2}$ for $4 m$ and $40.1 \mathrm{eV} / n^{2}$ for the $4 m+2$, respectively. Both converge to $E_{\infty}\left(\mathrm{sp}^{1}\right)=6.56 \mathrm{eV}$

The force field took the following form:

$$
\begin{aligned}
& E_{\mathrm{MSX}}=E_{0}+\sum_{l=1}^{N / 2}\left[\frac{1}{2} k_{1} q_{r 1}(l)^{2}\right. \\
& +\frac{1}{2} k_{2} q_{r 2}(l)^{2}+k_{12} q_{r 1}(l) q_{r 2}(l) \\
& \left.+k_{r r^{\prime}} q_{r 1}(l+1) q_{r 2}(l)\right]+\sum_{l=1}^{N} \frac{1}{2} k_{\theta} q_{\theta}(l)^{2} .
\end{aligned}
$$

Here $q_{r 1}(l)=R_{i}(l)-R_{i 0}(l)$ is the bond strain term, where for $n=4 m, i=1$ is the triple bond and $i=2$ is the single bonds; for $n=4 m+2$ their bonds are equivalent. The angle strain term is $q_{\theta}(l)=\theta(l)-\theta_{0}(l)$ We use the periodic boundary condition so that, with $\theta_{0}=\pi, n / 2+1=1$ where $n$ is the

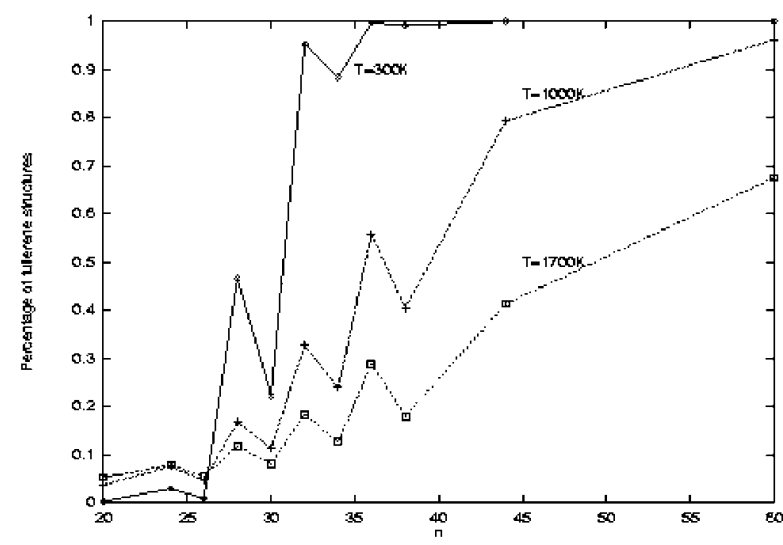

Figure 2. Population analysis of species.

total number of atoms in the system and $n / 2$ is the number of unit cells. $E_{0}$ is a reference energy corresponding to zero strain energy structure (infinite linear chain). Comparing $E_{\mathrm{MSX}}$ with $E_{\mathrm{DFT}}$ for several structures, we can derive the force field parameters.

In a similar fashion we can derive the force field for the $\mathrm{sp}^{2}$ bonded carbons. The optimum structure for bulk carbon is graphite, which has each carbon bonded to three others ( $\mathrm{sp}^{2}$ bonding) to form hexagonal sheets stacked on each other. The fullerenes structures can be considered as finite two-dimensional analogues, in which each carbon is distorted (strained) from its preferred planar configuration. Since the strain should be proportional to the square of the planar distortion angle, $\delta \psi$, we expect that the strain energy should scale as $1 / n$

We have performed the DFT(Becke/LYP) calculations on $\mathrm{C}_{n}$ fullerenes with $n=20,32$ and 60. Figure 1 shows the cohesive energies per carbon atom.

Extrapolating the calculated cohesive energy to $n \rightarrow \infty$ leads to a cohesive energy per $\mathrm{sp}^{2}$ carbon of $E_{\mathrm{coh}}\left(\mathrm{sp}^{2}\right)=$ $7.71 \mathrm{eV}$. This can be compared to the experimental cohesive energy of a single graphitic sheet of $E_{\text {coh }}^{\text {sheet }}=7.74 \mathrm{eV}$. This is derived from the experimental cohesive energy (CRC Handbook) of graphite of $E^{\text {graphite }}=7.8 \mathrm{eV}$ plus total Van der Waals attraction of $E^{\mathrm{vdw}}=0.056 \mathrm{eV}$ between sheets calculated using the graphite force field (Guo 1992).

Now that we have the energy and force field of both $\mathrm{sp}^{1}$ and $\mathrm{sp}^{2}$ hybridized carbon we can get the energetics of any carbon clusters. Adding the entropic contribution within the harmonic approximation using FF, we get the free energy of various species at different temperature, which dictates the thermal equilibrium distribution of these species. Our population analysis is shown in figure 2 .

For studying formation reaction sequence we adopt two level of models, a fine one and a coarse one, as explained below.

\subsection{Fine model}

The energies of the structures were computed via the following procedures that combine the DFT with MD as illustrated in figure 3.

(1) The reaction from two $\mathrm{C}_{30}$ ring (I) to $\mathrm{C}_{60}$ bicyclic ring molecules (II) is achieved via an intermediate III. For 

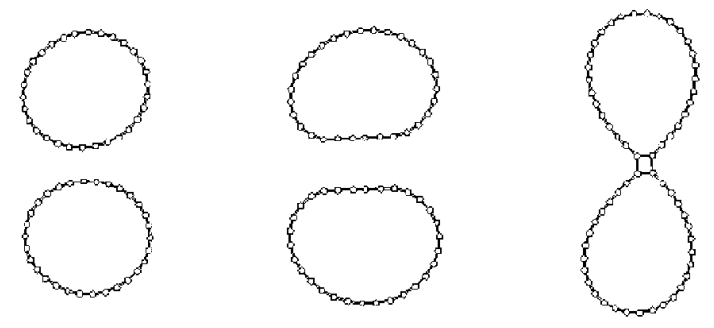

Figure 3. The reaction from two $\mathrm{C}_{30}$ ring to $\mathrm{C}_{60}$ bicyclic ring molecule.

each of I, II and III, the system is partitioned into two parts: part A involving bond lengths changes, and part B involving continuous deformation.

(2) The energy of I is determined directly from figure 1 .

(3) The energy difference between I and III is a strain energy which can be calculated using the MSX FF.

(4) The energy difference between III and II is calculated in two parts. (a) part A: DFT calculations are carried out on the reaction of two $\mathrm{C}_{6} \mathrm{H}_{2}$ molecules to form to the 4-membered ring, $\mathrm{C}_{12} \mathrm{H}_{2}$. (b) part $\mathrm{B}$ : we calculate the corresponding change in going from III to II using MSX FF. Then we combine A and B to get the energy difference between III and II.

(5) Thus the energy of $\mathrm{C}_{60}$ bicyclic ring (II) can be calculated by II-III-I.

\subsection{Coarse model}

We extend the MSX FF to include terms capable of describing the different bonding schemes. The key components are the additive energy terms for the dangling bond and the energy cost for bending a triple bond to form a 1,2-benzyne.

Our FF are defined as follows:

$$
\begin{aligned}
E_{\text {tot }}\left(n_{2}\right) & =E_{\text {bond }}+E_{\text {radical }}+E_{\text {strain }} \\
& =n_{2}\left(\epsilon_{1}-\epsilon_{2}\right)+d_{1} n_{R}+d_{2} n_{\sigma} \pi+E^{\mathrm{str}}\left(n_{2}\right) .
\end{aligned}
$$

We have chosen $E_{0}=60 \epsilon_{1}$, as zero point. Here, $n_{2}$ is the $\mathrm{sp}^{2}$ bonded carbons, $n_{2}\left(\epsilon_{1}-\epsilon_{2}\right)$ gives the energy gained by converting $\mathrm{sp}^{1}$ bonded carbon into $\mathrm{sp}^{2}$ bonded carbon, with $\epsilon_{1}=-6.56 \mathrm{eV}$ and $\epsilon_{2}=-7.71 \mathrm{eV} . d_{1}$ is the energy of a dangling bond relative to the $\sigma$-bonded state, $n_{R}$ number of such dangling bond(radicals); $d_{2}$ is the energy of an atom participating bended planar $\pi$-bond relative to the $\sigma$-bonded state and $n_{\sigma \pi}$ is the number of such atoms. We use the Benson-like scheme to evaluate $d_{1}$ and $d_{2}$ (Guo 1992) and found $d_{1}=2.32 \mathrm{eV}$ and $d_{2}=1.64 \mathrm{eV}$. $E^{\mathrm{str}}\left(n_{2}\right)$ is the strain energy and it is evaluated at the minimum energy structure.

We use the fine model for the initial steps in the $\mathrm{C}_{60}$ formation. As the reaction takes off and begins to release more and more energy, we switch to the coarse one.

\section{The spiral model of fullerene formation}

At the beginning, atomic carbons combine themselves to form dimers and trimer, $\mathrm{C}_{2}, \mathrm{C}_{3}$. These would then grow into linear chain of carbons $\mathrm{C}_{n}$, etc, for $n<10$ (Hutter et al 1994). When $n>10$ the carbon clusters prefer ring structure (Hutter et al 1994) because beyond $n>10$ the energy gain in killing
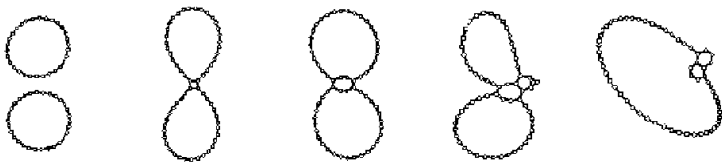

Figure 4. Illustration of the first few steps of reactions.

the dangling bonds at the two ends overcompensates for the strain energy incurred by folding up the chain. At around $n>30$ the ring structures give way to fullerene structures (von Helden et al 1993a, b) because replacing more $\pi$-bonds by $\sigma$-bonds overcompensates for the strain of folding the $2 \mathrm{D}$ net.

One process of $\mathrm{C}_{60}$ formation, as suggested by Jarrold's experiments (von Helden et al 1993a, b, Hunter et al 1994) is to combine two $\mathrm{C}_{30}$ rings to form a bicyclic $\mathrm{C}_{60}$ ring, which in turn is isomerized into a $\mathrm{C}_{60}$ fullerene. This unimolecular reaction will be the focus of our study.

As a mnemonic for referring to the various structures, we will simply denote the ring sizes of a structure. Thus the simple $\mathrm{C}_{60}$ ring is denoted as 60 , while the double ring system, 1 , is $30+4+30$. This notation does not uniquely describe a structure, but is for the species we will consider. We take the reference energy to be $E_{o}=60 \epsilon_{1}$, where $\epsilon_{1}=-6.56 \mathrm{eV}$.

Following Jarrold, the first few steps in the reaction are as follows (see figure 4):

(i) $1=\{30+4+30\} \rightarrow 2=\{30+4+6+30\}$. This is a Bergman diyne cyclization which forms a 6-membered 1,4 benzyne-like ring from two triple bonds. This leads to two isolated radical sites ( $\mathrm{sp}^{2}$-like orbitals in the plane, that cannot form a bond), and we find that this increases the energy by about $0.7 \mathrm{eV}$.

(ii) $2=\{30+4+6+30\} \rightarrow 3=\{30+8+30\}$. This process kills two dangling bonds by breaking one $\sigma$-bond and forming two $\pi$-bonds. This process is downhill by about $1.3 \mathrm{eV}$.

(iii) $3=\{30+8+30\} \rightarrow 4=\{30+8+6+22\}$. This involves breaking an in-plane $\pi$-bond and forming a $\sigma$ bond. In the process there is bending of one triple bond to form a 1,2-benzyne-like ring which includes a new radical site. This process is uphill by $1.66 \mathrm{eV}$. Jarrold postulated $4^{\prime}$ which is $2.1 \mathrm{eV}$ above the bicyclic rings from our calculation.

(iv) $4=\{30+8+6+22\} \rightarrow 5=\{6+6+55\}$. This involves twisting open the original 4 -membered ring. Then it is followed by relaxing the 50 carbon chain to reduce the strain energy. This $\{6+6+55\}$ contains two dangling bonds. This process is downhill by about $0.67 \mathrm{eV}$.

(v) Spiral growth around the $\{6+6\}$. As a first step $5=$ $\{6+6+55\} \rightarrow 6=\{6+6+53+5\}$. This uses one of the $\mathrm{sp}^{2}$ orbitals of the 1,2-benzyne-like ring to attack a triple bond and form a new 5-membered ring. This process is downhill by $0.13 \mathrm{eV}$.

(vi) Continue the spiral growth to form $\mathrm{C}_{60}$ fullerene. The energies calculated using the extended MSX FF on these systems are shown in figure 5 where we see that they are monotonically downhill. The overall gain of energy from $\{6+6+53+5\}$ to $\mathrm{C}_{60}$ is about $30 \mathrm{eV}$, so that no barriers are expected to impede these steps. Figure 6 


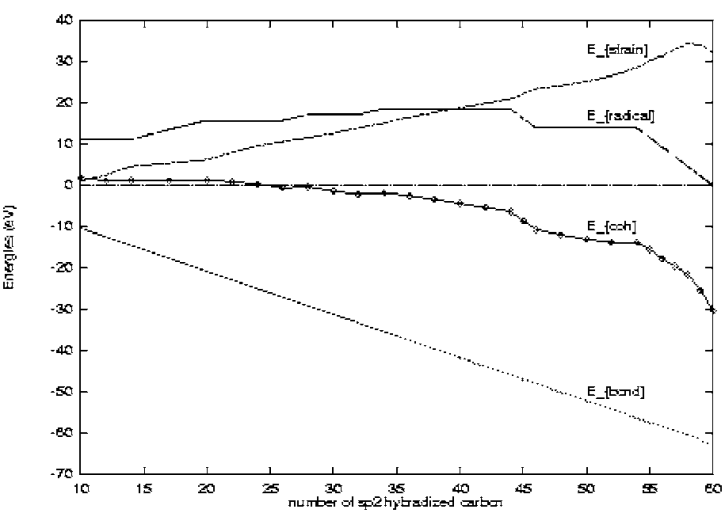

Figure 5. Energies calculated using the MSXX FF.<smiles>C1CCCCCCC1</smiles><smiles>C1CCCCCC1</smiles>
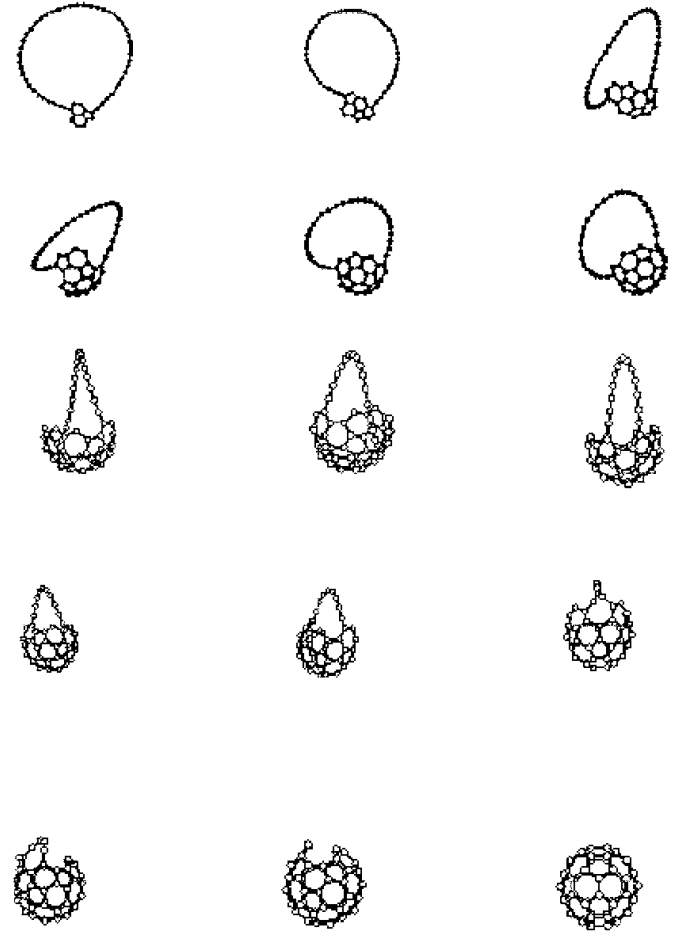

Figure 6. Intermediates between the 5 and the fullerene.

illustrates some of the intermediates between 5 and the fullerene.

The driving force for the growth is the gain in forming the $\operatorname{sp}^{2} \sigma$-bond. The opposing forces are the energy lost by the radicals created along the way and the increasing strain energies. The Jarrold mechanism represents an innovative major step forward in understanding the formation of $\mathrm{C}_{60}$ fullerene. Our energetic analysis shows that some of the reactions pathways have large energy barriers; however, they never exceed the energy available to the unimolecular reaction. A similar approach could be used to study the formation of other fullerenes, such as $\mathrm{C}_{40}, \mathrm{C}_{50}, \mathrm{C}_{70}$ etc.

\section{Summary}

Why $\mathrm{C}_{60}$ is so stable and how $\mathrm{C}_{60}$ fullerenes are formed, are the two most interesting problems in basic fullerene research. We have studied the formation mechanism of $\mathrm{C}_{60}$ fullerenes using first principles calculation and molecular dynamics simulations. We have derived a force field (MSX FF) that is suitable to describe both the $\mathrm{sp}^{1}$ hybrid and $\mathrm{sp}^{2}$ hybrid carbons. Combining DFT and MD with MSX FF we found the relative thermal stability of various neutral isomers at each cluster size $n$ and predicted the relative abundance of these neutral species for thermal equilibrium. We have identified a complete path to form a $\mathrm{C}_{60}$ fullerene from atomic carbons and calculated its energetics. Our approach is fully applicable to other possible reaction paths and other fullerenes.

\section{Acknowledgments}

This work is supported by computational nanotechnology grant from NASA Ames. The facilities of MSC is also supported by funds from NSF (CHE 95-22179), DOE-ASCI, NASA/Ames, Avery Dennison, BP Chemical, Beckman Institute, Chevron Petroleum Technology Co., Chevron Chemical Co., Exxon, Dow Chemical and SeikoEpson.

\section{References}

CRC Handbook

Feyereisen M, Gutowski M, Simons J and Alm J 1992 Relative stabilities of fullerene, cumulene and polyacetylene for structures $\mathrm{C}_{n} n=18-60 \mathrm{~J}$. Chem. Phys. 96 2926-32

Guo Y 1992 PhD Thesis California Institute of Technology

Hunter J M, Fye J L and Jarrold M F 1993 Annealing and dissociation of carbon rings J. Chem. Phys. 99 1785-95

Hunter J M, Fye J L, Roskamp E J and Jarrold M F 1994 Annealing carbon cluster ions-A mechanism for fullerene synthesis J. Phys. Chem. 98 1810-8

Hutter J, Luthi H P and Diederich F 1994 Structures and vibrational frequencies of the carbon molecules $\mathrm{C}_{2}-\mathrm{C}_{18}$ calculated by density functional theory J. Am. Chem. Soc. 116 $750-6$

Johnson B G, Gill P M W and Pople J A 1993 The performance of a family of density functional methods J. Chem. Phys. 98 $5612-26$

Konig G and Stollhoff G 1990 Why polyacetylene dimerizes-results of ab initio computations Phys. Rev. Lett. 65 1239-342

Raghavachari K, Strout D L, Odom G K, Scuseria G E, Pople J A, Johnson B G and Gill P M W 1993 Isomers of $C_{20}$-dramatic effect of gradient corrections in density functional theory Chem. Phys. Lett. 214 357-61

Rignalda M N et al 1995 PS-GVB v2.2 (Schrodinger, Inc.) von Helden G, Gotts N and Bowers M T 1993a Experimental evidence for the formation of fullerenes by collisional heating of carbon rings in the gas phase Nature $\mathbf{3 6 3} 60-3$

von Helden G, Hsu M-T, Gotts N and Bowers M T 1993b Carbon cluster cations with upto 84 atoms - structures, formation mechanisms and reactivity J. Phys. Chem. 97 8182-92 